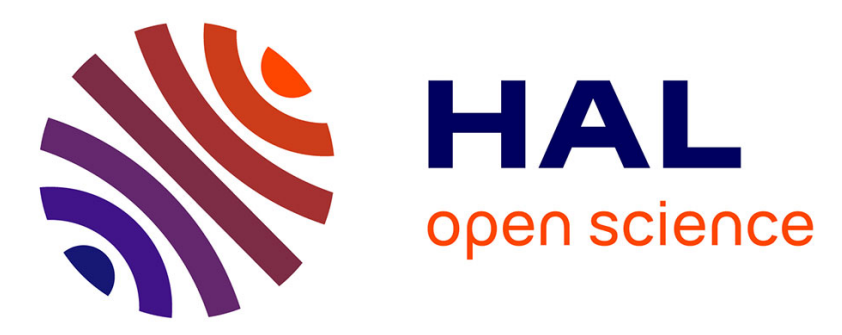

\title{
Le fonds Montet et la statue " maussade" de Ramsès II au Palais Universitaire de Strasbourg
}

Cassandre Hartenstein

\section{To cite this version:}

Cassandre Hartenstein. Le fonds Montet et la statue "maussade" de Ramsès II au Palais Universitaire de Strasbourg. Archimède: archéologie et histoire ancienne, 2015, 2, pp.41-50. halshs-01587278

\section{HAL Id: halshs-01587278 \\ https://shs.hal.science/halshs-01587278}

Submitted on 14 Sep 2017

HAL is a multi-disciplinary open access archive for the deposit and dissemination of scientific research documents, whether they are published or not. The documents may come from teaching and research institutions in France or abroad, or from public or private research centers.
L'archive ouverte pluridisciplinaire HAL, est destinée au dépôt et à la diffusion de documents scientifiques de niveau recherche, publiés ou non, émanant des établissements d'enseignement et de recherche français ou étrangers, des laboratoires publics ou privés. 


\section{ARCHIMĖDE N $N^{\circ} 2$}

\section{DOSSIER THÉMATIQUE : ARCHIVES DE L'ARCHÉOLOGIE}

1 Marie STAHL

Introduction : la mémoire retrouvée des archéologues

9 Marie STAHL, Lucile SCHIRR

Les archives de l'archéologie : définition, législation, état des lieux

20 Dominique BEYER, Marie STAHL (collab. Catherine DUVETTE, Isabelle WEYGAND, Françoise LAROCHE-TRAUNECKER, Marie-José MORANT, Philippe QUENET) Les archives de la composante d'archéologie orientale de I'UMR 7044

\section{Cassandre HARTENSTEIN} Le fonds Montet et la statue « maussade » de Ramsès II au Palais universitaire de Strasbourg

51 Soline MORINIÈRE

Les archives de l'archéologie au SRA Alsace : état des lieux et des fonds

59 Anne ROHFRITSCH

Les archives dites « manuscrites » des membres de l'École française d'Athènes :

l'exemple des « Strasbourgeois 》 (1846-1960)

66 Cécile COURTAUD, Isabelle LESUEUR, Soline MORINIÈRE, Juliette RÉMY, Bernadette SCHNITZLER, Marie STAHL, Georges TRIANTAFILLIDIS Un projet collectif de recherche autour du fonds Arthur Stieber

78 Soline MORINIÈRE

La gypsothèque de I'Université de Strasbourg : quand les statues parlent d'elles-mêmes

\section{LA CHRONIQUE D'ARCHIMÈDE}

94 Frédéric COLIN (éd.)

La Chronique d'Archimède. Bilan des activités scientifiques 2014-2015 de I'unité mixte de recherche 7044

\section{VARIA}

134 Sarah DERMECH

Couleurs, éclat et brillance des crânes surmodelés : le cas du Néolithique Proche-oriental

150 Cinzia BEARZOT

La violence de l'État. La condamnation à mort sans jugement dans la Grèce ancienne

160 Doris MEYER

Jusqu'au dernier mot. Martyr, débat public et résistance dans la littérature de I'Antiquité tardive et à Byzance

170 Vincent PUECH

Les biens fonciers des élites sénatoriales à Constantinople et dans ses environs (451-641)

194 Clara MILLOT

Entre les enfants d'Hérodote et les enfants d'Adam Smith. Pour une approche économique des données archéologiques

Retrouvez tous les articles de la revue ARCHIMĖDE sur :

http://archimede.unistra.fr/revue-archimede/archimede-2-2015 


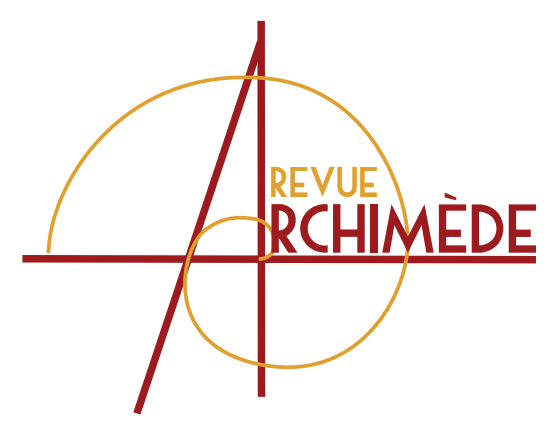

\title{
LE FONDS MONTET ET LA STATUE « MAUSSADE » DE RAMSÈS II AU PALAIS UNIVERSITAIRE DE STRASBOURG
}

\author{
Cassandre HARTENSTEIN \\ Doctorante, Université de Strasbourg, \\ UMR 7044 Archimède \\ hcassandre@gmail.com
}

RÉSUMÉ

L'Institut d'égyptologie de I'Université de Strasbourg possède une collection d'environ 5000 négatifs et positifs photographiques sur verre datant de la fin du $\mathrm{XIX}^{\mathrm{e}}$ siècle jusqu'aux années 1950. Un certain nombre d'entre eux (à peu près 900) sont issus des fouilles menées par Pierre Montet dans le Delta du Nil, à Tanis, depuis 1929 jusqu'en 1952. Ces plaques de verre comportant des clichés d'objets découvertsetdesétatsd'avancementdecesexcavations, ont été récemment inventoriées et reconditionnées. L'objectif de cet article est de présenter cet ensemble d'archives d'une fouille française en Égypte et de montrer l'intérêt de lancer une étude croisant les informations fournies par ce fonds avec les objets actuellement conservés à I'Institut d'égyptologie de Strasbourg. En effet, la provenance d'un grand nombre de ces artefacts est inconnue; les archives Montet pourraient permettre de reconstituer un contexte

Mots-CLÉs

Tanis,

archives,

plaque photographique,

Ramsès II

collection d'objets de l'Institut

d'Égyptologie de I'Université de

Strasbourg.

\begin{abstract}
Je tiens à remercier chaleureusement Frédéric Colin pour avoir relu ce texte et réalisé les clichés des plaques de verre. Ma reconnaissance va également à François Leclère et Patrice Le Guilloux, qui m'ont aimablement fourni des informations importantes notamment pour les légendes des plaques photographiques.
\end{abstract}

The Institute of Egyptology of Strasbourg's University owns a collection of about 5000 negatives and positives on glass, dated from the end of the XIX'th century to the 1950s. Many of them (about 900) come from excavations lead by Pierre Montet in Tanis, which took place from 1929 to 1952 . Those glass plates have pictures of objects found and of the progression of the excavations. They have been inventoried and repackaged recently. This paper aims at presenting this group of archives from Egyptian excavations and at showing the interest of starting a study crossing the informations from this fund with the pieces currently located at the Institute of Egyptology of Strasbourg's University. Indeed, the origin of many of the objects is unknown; the Montet's Archives could make it possible to understand the archaeological conKeywords Tanis, archives, glass plate, Ramsès II, collection of objects Institute of Egyptology of Strasbourg's University 
Durant les trente années qu'il passa à la tête de I'Institut d'égyptologie de Strasbourg [1], Pierre Montet dirigea deux chantiers de fouille, à Byblos [2] et à Tanis, site qu'il rendit célèbre lors de la découverte d'une nécropole royale en 1939 [3]. De ses excavations à Byblos, P. Montet a laissé à I'Université de Strasbourg une vingtaine de négatifs photographiques sur plaques de verre. Il a également ramené de Tanis une collection de négatifs et positifs sur plaques de verre, et des objets issus de partages entre les États égyptien et français de 1929 à 1939. Quelques archives sur support papier [4] (dessins, estampages) issues des fouilles ont été déposées à I'Institut strasbourgeois. Les archives publiques de la «Mission Montet» se partagent aujourd'hui entre Strasbourg et Paris [5], d'où P. Montet poursuivit ses fouilles, jusqu'en 1956. Après une courte période durant laquelle ces fouilles ont été interrompues, Jean Yoyotte créa en 1965 la Mission française des fouilles de Tanis [6]. C'est ainsi qu'après sa nomination, ce savant annonça son intention de réunir et de dresser l'inventaire de la documentation issue de la Mission de Tanis dans une lettre adressée à Philippe Derchain, alors chargé de cours à I'Institut d'égyptologie de Strasbourg [7] : « Un aspect rebutant de la Mission de Tanis est pour moi la perspective d'avoir à reconstituer les archives de la Mission. S'il y a tant soit peu quelque chose (objets, documents) de Tanis à Strasbourg, aie la bonté de m'en informer. Comme j'aimerais me rendre dans votre ville d'ici l'été, pourrais-tu me dire du même coup jusqu'à quelle date tu comptes y résider avant de partir en vacances. » $\mathrm{Ph}$. Derchain répondit favorablement à la demande qui lui était faite, puisque J. Yoyotte lui transmit en octobre de la même année [8] un texte listant les différents lieux où étaient conservés les documents de la Mission Montet, dont Strasbourg faisait partie. Le document indique que la Faculté des Lettres de Strasbourg était en possession de photographies collées sur cartons [9], de dessins et de négatifs photographiques sur plaques de verre. Un inventaire plus précis des archives strasbourgeoises est établi en juin de I'année suivante par J. Yoyotte, au moment où elles sont provisoirement confiées en dépôt à I'École Pratique des Hautes Études (EPHE) [10].

Depuis 2010, I'Institut d'égyptologie de Strasbourg a entrepris une opération de reconditionnement [11] des plaques photographiques qui y sont conservées, avec I'aide d'étudiants vacataires et stagiaires [12]. Les plaques sont à présent conservées dans des pochettes individuelles [13]. En plus des négatifs de la Mission de Tanis, la collection comporte des négatifs de vues de différents sites égyptiens et un lot important de positifs sur verre commandés par W. Spiegelberg (environ 3000 pièces). Les plaques de verre provenant des fouilles de Tanis sont
[1] À partir de 1919, Pierre Montet fut chargé de cours à I'Université de Strasbourg. Nommé professeur en 1934, il quittera I'Alsace pour le Collège de France en 1948.

[2] Le travail de P. Montet à Byblos s'étale sur quatre années (1920-1924).

[3] Sur les travaux de P. Montet à Tanis, voir notamment MONTET 1933a; MONTET 1942. Voir également les rapports de P. Montet dans les Bulletins de la Faculté des Lettres de Strasbourg de 1928 à 1939. Pour une vue d'ensemble sur les fouilles de Tanis depuis le XIXe siècle, voir BRISSAUD, CHAUVET \& HAIRY, 1998, p. 71-100 et LECLÈrE 2006, p. 397-400.

[4] Les archives et les objets issus des fouilles de P. Montet présentés dans cet article sont aujourd'hui conservés à la MISHA (Maison Interuniversitaire des Sciences de I'Homme-Alsace) à Strasbourg. En plus de la collection de plaques de verre des objets tanites, P. Montet aurait laissé à l'Institut d'égyptologie de Strasbourg 14 estampages et 36 dessins et calques de L. Épron, qui n'ont pas été localisés pour le moment (voir la lettre de J. Yoyotte à Ph. Derchain du 20 juin 1966).

[5] Le Guilloux 1998, p. 179-192.

[6] YoYotTe 1965, p. 392 ; LeCLÈre 2006, p. 399.

[7] Lettre datée du $1^{\text {er }}$ juin 1965 (Archives de l'Institut d'égyptologie de Strasbourg).
[8] Lettre du 23 octobre 1965 (Archives de I'Institut d'égyptologie de Strasbourg).

[9] Ces tirages papier prêtés à I'EPHE I'année suivante sont en fait des photographies des campagnes de P. Montet à Abou Roach, et non de Tanis.

[10] Lettre du 20 juin 1966 (Archives de I'Institut d'égyptologie de Strasbourg). Les archives de I'Institut d'égyptologie ne donnent aucune information sur la date à laquelle les documents ont été retournés à Strasbourg.

[11] Un inventaire a été réalisé en 2004 par A. Schweitzer, avec I'aide de S. Zanatta et A. Gräzer.

[12] Ont participé à cette opération : outre l'auteur (responsable de l'opération), Saber Hassan Ramadan (2011 et 2014); Simon Thault (2012); Alexandre Freund-Lehmann (2012); Vincent Gauthier (2013). À ce jour, une centaine de plaques n'ont pas encore été inventoriées et reconditionnées. Ce projet a pu être mené grâce au soutien du Jardin des Sciences de I'Université de Strasbourg.

[13] Le reconditionnement des plaques de verre a été effectué en suivant les instructions proposées par le musée municipal George Tupin de Parthenay (www.alienor.org/publications/plaque_verre/texte_a_imprimer.htm, site consulté le $13 / 11 / 2014)$. 
de format $18 \times 24 \mathrm{~cm}$ [14], $9 \times 12 \mathrm{~cm}$ et $8,5 \times 10 \mathrm{~cm}$ et forment un ensemble de 901 pièces [15]. Les plaques des deux premiers formats sont des négatifs, alors que celles de $8,5 \times 10 \mathrm{~cm}$ sont des positifs. Les négatifs ont été inventoriés, probablement par l'équipe de J. Yoyotte, puisque chaque pièce comporte une référence I.E.S. TN, suivi du numéro de la boîte dans laquelle elle se trouve, et du numéro de sa position dans la boîte. Cette procédure pour référencer les plaques a été proposée par J. Yoyotte à $\mathrm{Ph}$. Derchain dans une lettre conservée à I'Institut d'égyptologie. Contrairement aux plaques de format $18 \times 24 \mathrm{~cm}$ et $9 \times 12 \mathrm{~cm}$, les $8,5 \times 10 \mathrm{~cm}$ ne sont pas marquées d'un numéro d'inventaire (ce qui rend leur identification plus difficile, puisqu'elles sont mélangées à des prises de vues d'autres sites et d'objets n'ayant rien à voir avec Tanis). Ces plaques n'apparaissent par ailleurs pas dans la liste officielle des archives déposées pour étude à I'EPHE en 1966 (voir note 10), ce qui explique pourquoi elles ne sont pas numérotées comme les négatifs [16]. La base de données réalisée sous la direction d'A. Schweitzer (http://www2i.misha.fr/flora/servlet/ LoginServlet) signale que des plaques de format 8,5 $\mathrm{x}$ $10 \mathrm{~cm}$ réalisées à l'époque allemande contiennent des vues de Tanis ou des objets provenant des fouilles de Mariette réalisées dans cette ville et conservées au Musée du Caire. Leur nombre est inconnu à ce jour. Les sujets des plaques réalisées par $P$. Montet se divisent en deux catégories : les clichés immortalisant des scènes de fouilles ou l'état d'avancement de secteurs (fig. 1), et des photographies d'objets (fig. 2). Sur ces dernières, les objets sont disposés soit sur des fonds uniformes (couvertures disposées sur le sol ?), soit sur un dallage ou encore sur



[14] On compte 435 plaques de format $18 \times 24 \mathrm{~cm}, 417$ de dimensions $9 \times 12 \mathrm{~cm}$.

[15] J. Yoyotte a noté dans son inventaire du 20 juin 1966 que « certaines plaques sont cassées $\gg$. L'inventaire nous a permis de relever 4 plaques brisées et 6 manquantes.

[16] Après un premier examen, il semblerait que les plaques positives soient des diapositives, tirées d'après des négatifs sur verre, qui sont dans certains cas conservés à Strasbourg ( $\mathrm{Fr}$. Leclère $\mathrm{m}$ 'indique que des plaques de même format se trouvent à I'EPHE, et qu'elles étaient utilisées par $\mathrm{P}$. Montet pour ces cours et conférences). Les négatifs des diapositives qui n'ont pas été trouvés sont peut-être conservés à I'EPHE.
Figure 1 : IES Tn 32/12

Vue de la nécropole royale en cours de fouille, avec au premier plan la tombe d'Osrokon II et au centre celle de Psousénnès. Ce cliché a probablement été réalisé en 1939, année de la découverte de la nécropole royale. 
Figure 2 : IES Tn 10/8

Ce cliché semble avoir été pris à Tanis en 1939, par Georges Goyon, comme l'indique une mention manuscrite de Jean Yoyotte présente sur l'Album MM 67, dans lequel se trouve un tirage papier de ce négatif. II s'agit des parures trouvées dans l'antichambre de NRT III le 17 mars 1939, sur l'un des squelettes reposant de part et d'autre du cercueil d'argent de Chéchanq II, identifiés par J. Yoyotte comme étant ceux de Psousennès II et Siamon.

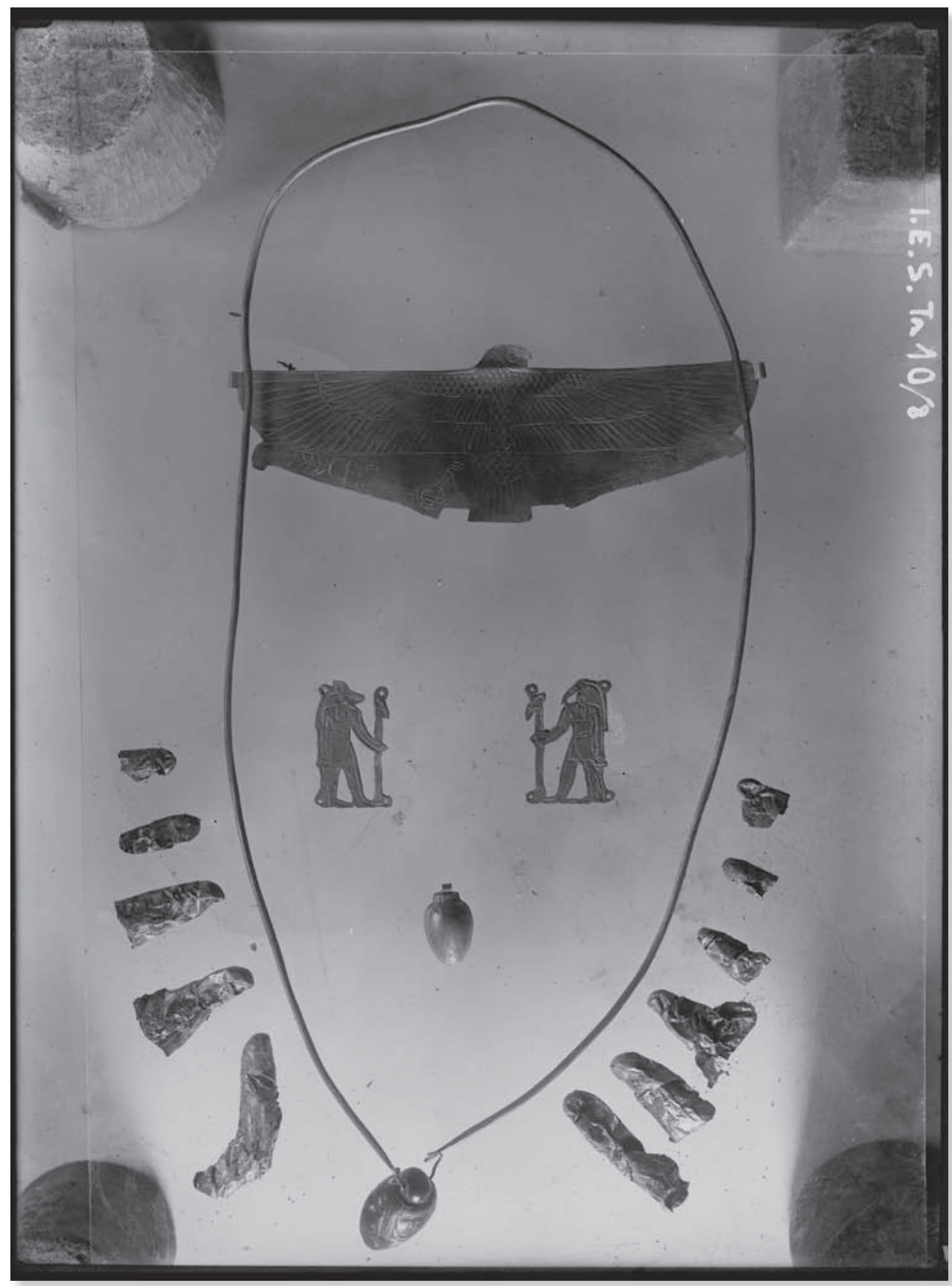

des étagères, peut-être celles qui se trouvaient dans la maison de fouilles de la Mission [17].

En plus de cette importante collection de clichés de Tanis, P. Montet enrichit la collection égyptienne d'objets issus de ses fouilles dans le Delta, dont le nombre est encore aujourd'hui inconnu. Le cahier d'inventaire de l'Institut ne comporte pas de section où sont listés les objets provenant de Tanis, contrairement à ce qui a été fait pour le produit des fouilles d'Abou Roach. Les seules pièces au sujet desquelles nous savons avec certitude qu'elles provenaient de Tanis sont celles qui ont été publiées par P. Montet avec une photographie [18]. À ce jour, seules onze pièces ont été retrouvées depuis le début du nouvel inventaire de la collection de Strasbourg en 2007, d'après des photographies [19]. Aucun de ces objets ne porte un numéro qui aurait pu être attribué lors des fouilles ou lors des partages entre musées égyptien et français, ce qui aurait aidé à repérer les objets de Tanis dans la collection strasbourgeoise.

[17] Parmi ses souvenirs de Tanis, Camille Montet-Beaucour se rappelle qu'un magasin est construit à côté de la maison de fouilles. Les étagères visibles sur les négatifs sont peutêtre celles de ce bâtiment, dans lequel les objets découverts étaient entreposés (MONTET 1998, p. 26).

[18] P. Montet a publié dans plusieurs travaux des clichés d'objets déposés dans la collection strasbourgeoise : MONTET 1933a, PI. LXI, LXII, LXIV, LXV; MONTET 1942, p. 190.

[19] IES NI 0111, IES NI 0353, IES NI 0769, IES NI 0871, IES_NI_1026, IES_NI_5097, IES_NI_5160, IES_NI_5180, IES_NI_5205, IES_NI_5236. 
Parmi l'ensemble des négatifs sur verre, se trouve un lot de vues de deux statues de Ramsès II, retrouvées dans le vestibule du temple dit d'Anta [20]. P. Montet en fait une description dans une publication des fouilles de Tanis [21] : «... nous avons pu mettre la main sur trois des statues que Ramsès y avait fait déposer. Deux de ces statues représentent le roi assis sur un siège carré, plein, à dossier bas, impassible, les mains appliquées sur les genoux. Il est coiffé du nems; les yeux sont fardés ; deux attaches qui partent du bandeau frontal supportent la barbe postiche qui laisse apercevoir entre les deux retombées du nems quelques perles du gorgerin. Le pagne plissé très simple est tenu par une ceinture dont la boucle est gravée au nom de Ramsès. La mieux conservée des deux statues ne présente que des mutilations superficielles: I'uraeus, le nez, les doigts de pied. Elle est aussi la plus séduisante. L'autre est plus maussade, ce qui tient peut-être aux mutilations du nez, du menton, des mains et des genoux, mais aussi aux plis trop accentués des lèvres et aux yeux trop à fleur de tête, comme sur les bas-reliefs du temple et sur la stèle de I'an 400. » Grâce à cette description, il est facile de différencier les deux statues sur les plaques de verre : la statue « séduisante » se trouve sur les plaques I.E.S. TN 11/7 [22] (fig. 3), TN 11/8 [23] (fig. 4), TN 11/1 [24] (fig. 5) et TN 13/3 [25] (fig. 6) et la « maussade » sur les clichés I.E.S. TN 11/3 (fig. 7) et I.E.S. TN 11/5 [26] (fig. 8). La seconde statue, la «maussade » selon les

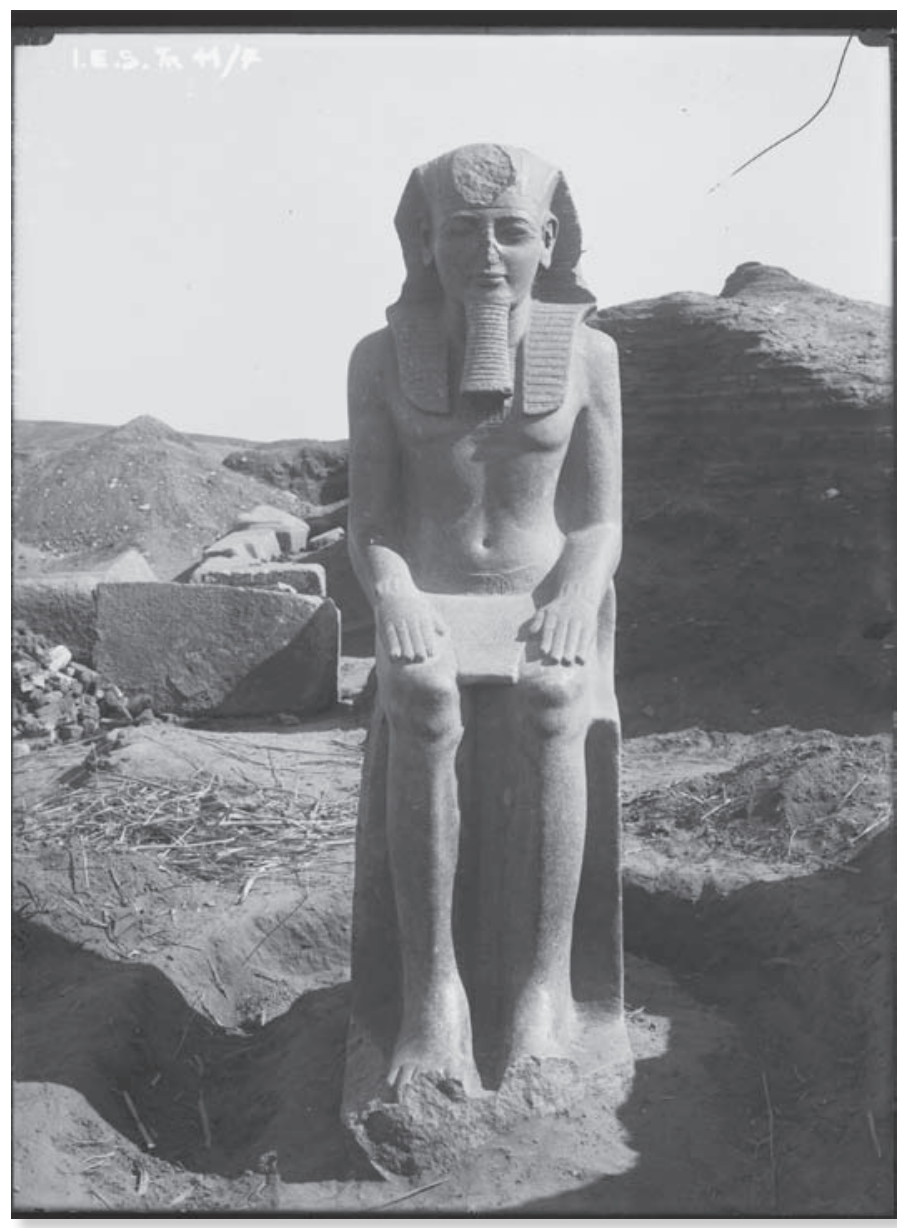

[20] Sur la découverte de ces deux statues voir, MoNTET 1933, p. $2-8$.

[20] Le temple dit d'Anta a été fouillé par Ph. Brissaud, qui le nomme aujourd'hui «temple de Mout et Khonsou l'enfant ». Au sujet de ces nouvelles données, voir BRISSAUD 2011 et BRISSAUd \& DESBORDES 2012.

[21] MONTET 1942, p. 193-194. Hormis cette description et une photographie publiée dans MonTEt 1935-1938, p. 498-508, PI. I, $n^{\circ} 4$, P. Montet n'a publié aucune information sur cette statue.

[22] $P$. Montet a fait faire des tirages papiers d'un certain nombre de plaques de verre, qu'il a ensuite placé dans des

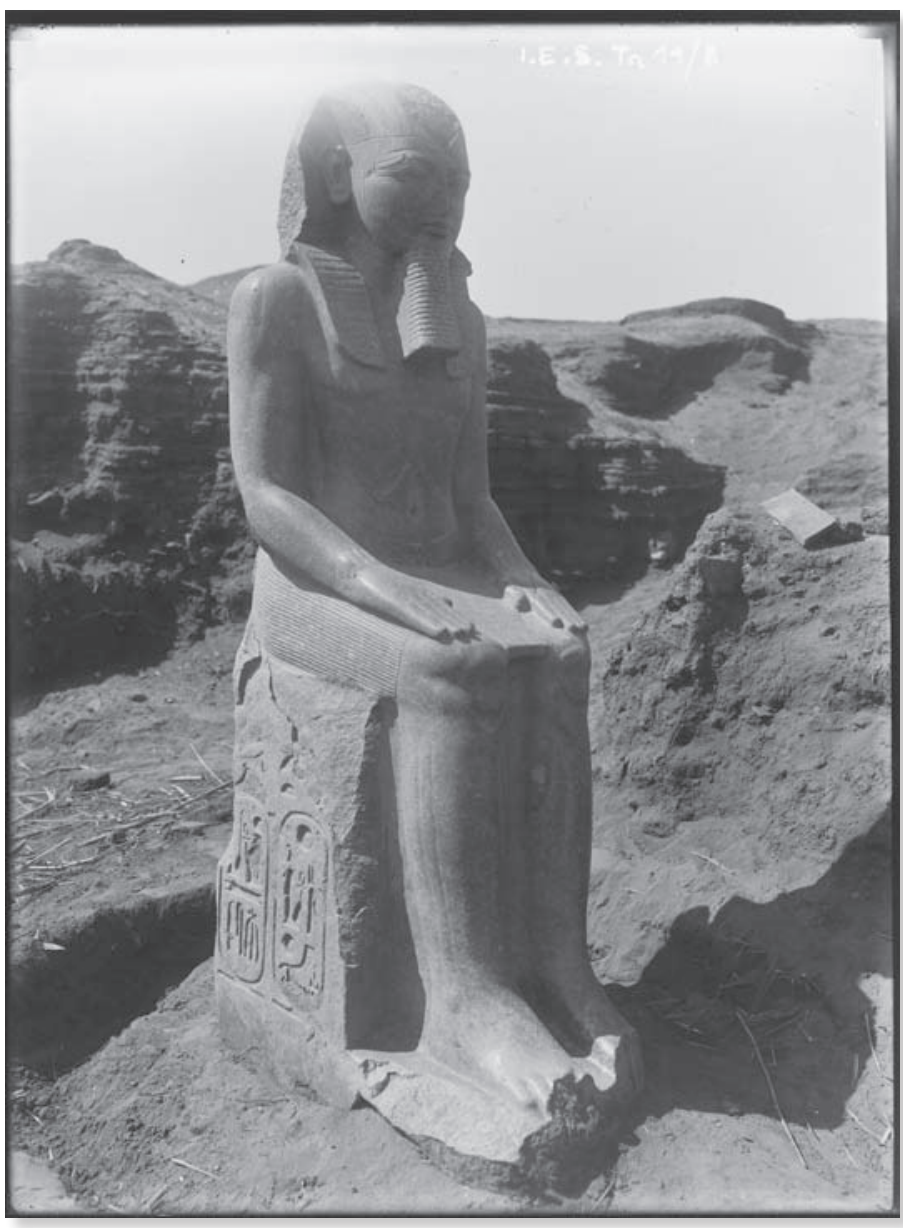

albums photographiques, aujourd'hui conservés au centre Goelenischeff de I'EPHE. Lorsqu'une version positive d'une plaque strasbourgeoise figure dans I'un de ces albums, j'indiquerai en note la référence en question. La plaque TN 11/7 correspond au tirage papier Alb. MM 14, 51. Je remercie P. Le Guilloux pour ces informations.

[23] Alb. MM 14, 52.

[24] Alb. MM 13, 33.

[25] Alb. MM 15, 28

[26] Alb. MM 13, 35 


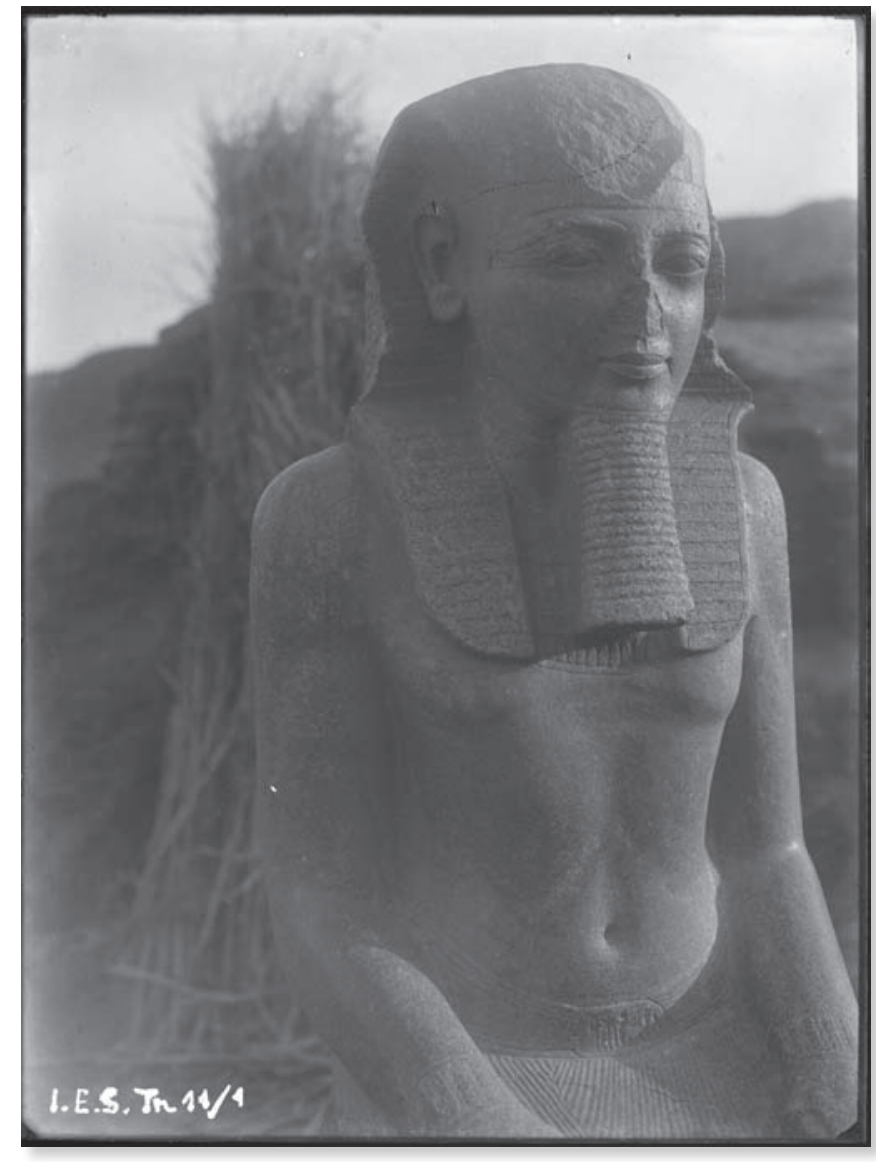

Figure 5 : IES Tn 11/1

Gros plan de la statue " séduisante » de Ramsès II découverte dans le temple dit d'Anta en 1933, au moment de sa découverte.

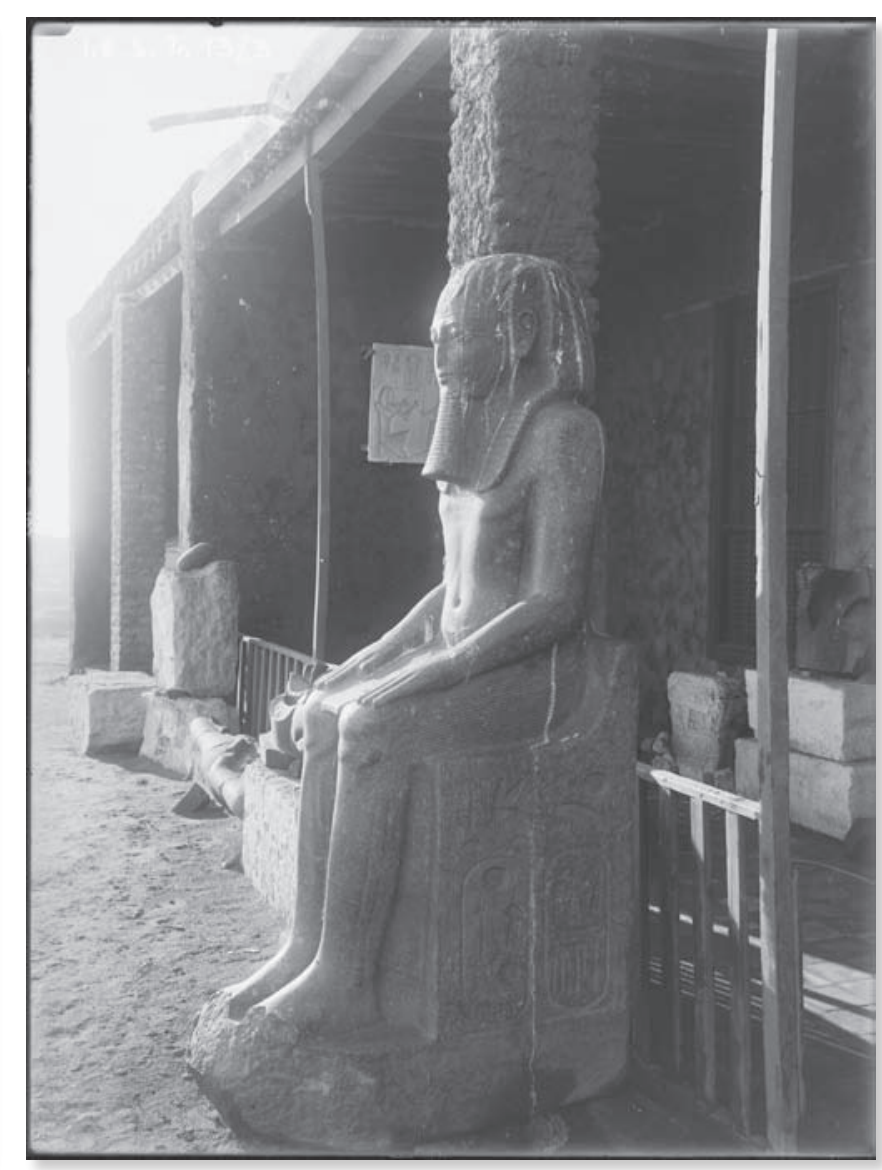

Figure 6 : IES Tn $13 / 3$

Statue « séduisante » de Ramsès II installée devant la maison des fouilles de Tanis.

Figure 8 : IES Tn 11/5

Statue « maussade » de Ramsès II au moment de sa découverte (aujourd'hui à Strasbourg) avec un ouvrier.
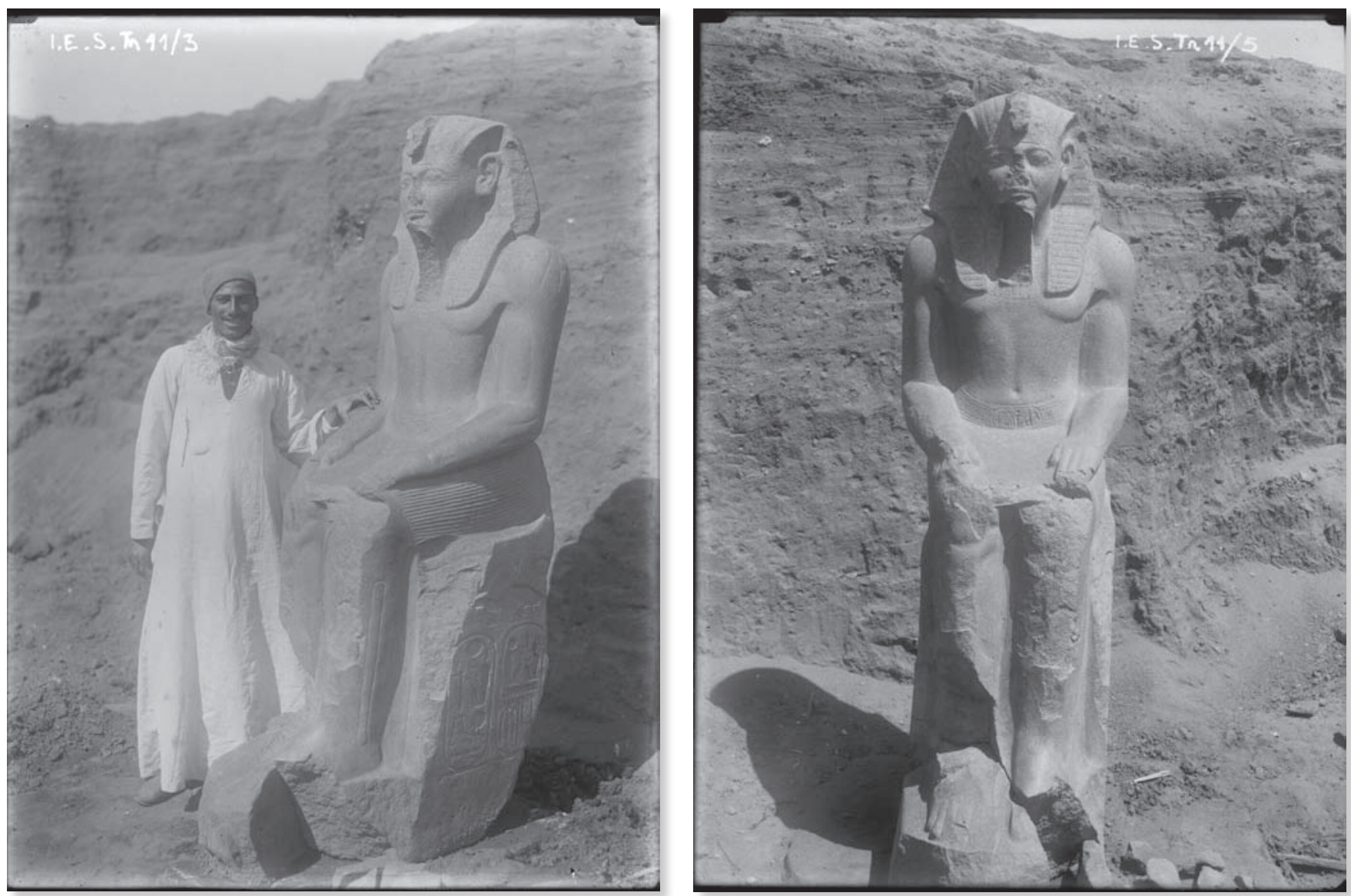
qualificatifs esthétisants de P. Montet, photographiée in situ lors de sa découverte, est sans aucun doute celle qui se trouve aujourd'hui dans I'aula [27] du Palais de I'Université de Strasbourg (fig. 9) [28]. Cette identification est certaine d'après les similitudes et les détériorations subies (barbe postiche disparue, pied gauche manquant) [29]. Découvert en 1933 [30], le Ramsès « maussade » est resté cinq ans à Tanis, avant d'être envoyé en France, comme cadeau de l'Égypte à la France [31]. Dans la lettre qui suit, P. Montet sollicite le doyen de la Faculté des Lettres de Strasbourg, afin d'obtenir le financement complémentaire qui lui permettrait de faire venir la statue à Strasbourg.

«Strasbourg, le 6 janvier 1938

Monsieur le Doyen,

J'ai I'honneur de vous faire savoir que le gouvernement égyptien a attribué à la France une statue de Ramsès II, en granit, haute de 2 mètres, découverte par la mission

Figure 9a

Statue « maussade » de Ramsès II aujourd'hui au Palais Universitaire (@ Pascal Disdier/CNRS)

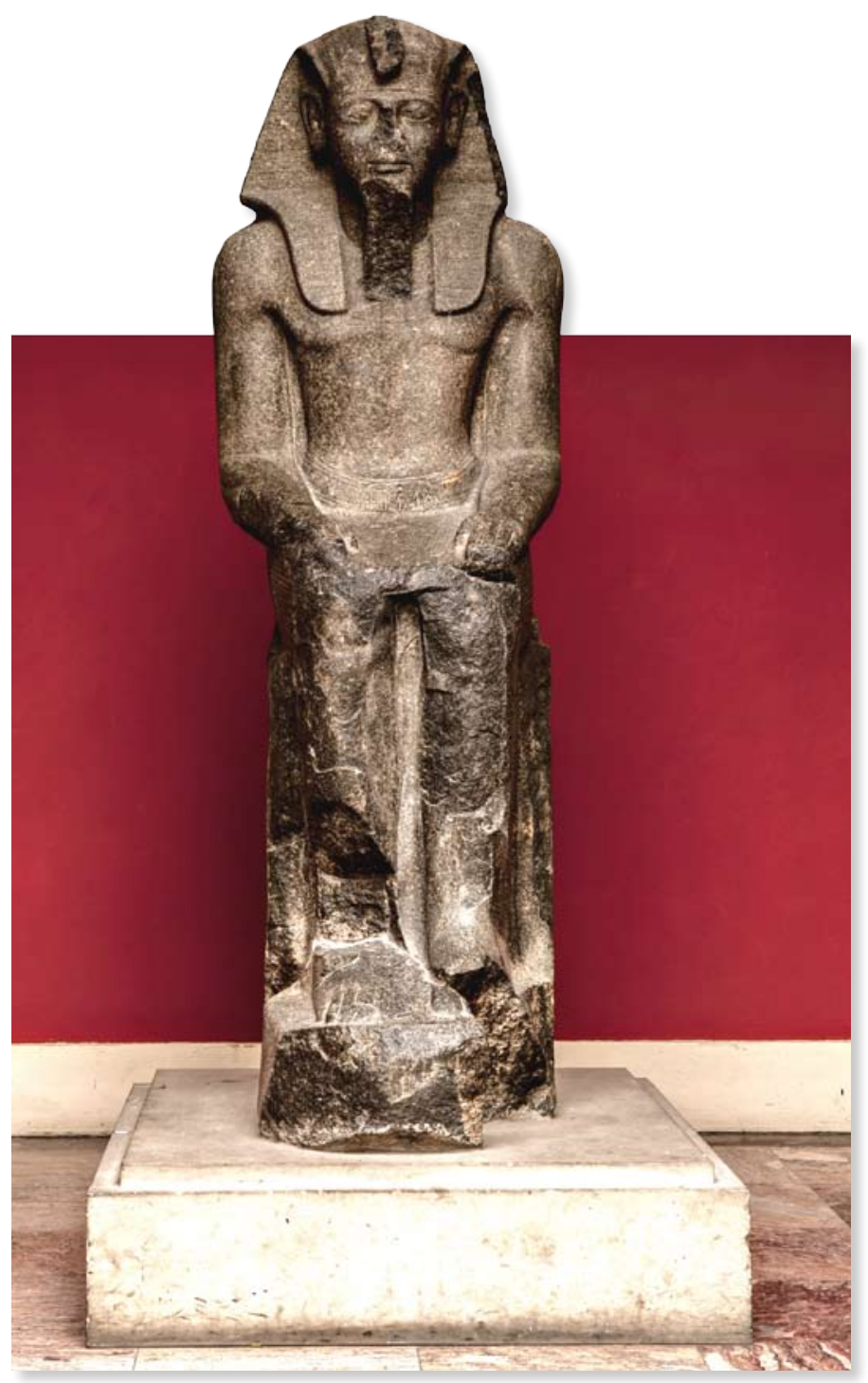

de Tanis. La Commission ministérielle des fouilles de Tanis m'autorise à faire transporter cette statue au Musée égyptologique du Palais du Rhin, mais elle ne peut se charger des frais de transport, qui d'après le devis ci-joint de la compagnie Cook, atteindrait environ 3.000 frcs, et même le dépasserait d'environ 1.000 frcs, si I'on joint à la statue une stèle de la IVème dynastie et un petit sarcophage de pierre que j'ai découverts près des pyramides en 1913 et qui, attribués à la France, sont restés dans les réserves de I'Institut français.

C'est donc une dépense de 4.000 frcs à 5.000 frcs qu'il faut prévoir. Je puis y consacrer le reliquat de la Société des Amis de Tanis qui se monte à 1500 frcs. Restent à trouver 3500 frcs.

Permettez-moi de faire remarquer que la valeur marchande des pièces archéologiques énumérées plus haut dépasse certainement cent mille francs et que le Gouvernement égyptien interdira, dit-on, dans un avenir peu éloigné, toute sortie d'objet antique.

Je me permets donc d'espérer que cette occasion d'augmenter la valeur de notre collection du Palais du Rhin ne sera pas négligée.

Veuillez agréer, Monsieur le Doyen, mes sentiments respectueux,

Pierre Montet »[32].

La somme sera versée par les autorités de l'université le mois suivant pour acheminer la statue vers Strasbourg. Le périple depuis Tanis en passant par Port-Saïd et Anvers, est décrit par P. Montet dans le Bulletin de la Faculté des Lettres de Strasbourg [33]. P. Montet souhaitait que

[27] Le nom d'« aula », employé couramment pour désigner l'espace central du Palais Universitaire de Strasbourg, est en réalité impropre. Au moment de la construction du bâtiment, I'actuelle salle Pasteur était nommée «aula » et l'emplacement actuel de la statue de Ramsès était la «cour intérieure du Palais » (conférence d'H. Doucet, « Histoire du Palais universitaire », Strasbourg, le 17 septembre 2014).

[28] La première statue a sans doute été installée après sa découverte devant la maison de fouilles de Tanis. Si on compare les plaques de verre et la photo publiée par C. Montet Beaucour (MoNTET 1998, fig. 5), où les trois filles de $P$. Montet entourent une statue de Ramsès II, il ne fait aucun doute qu'il s'agit du même colosse. Dans l'introduction de l'ouvrage cité ci-dessus, C. Montet-Beaucour indique que la statue qui décorait l'entrée de la maison de fouilles est actuellement au Caire, sur l'île de Gezirah (op. cit., p. 25).

[29] Cette statue est inconnue du P\&M. Une publication est en cours de préparation.

[30] MONTET 1933b, p. 2-8.

[31] Les deux statues de Ramsès font partie des objets partagés en 1937 entre l'Égypte et la France. Selon le document de partage établi à l'issue de la fouille, le Ramsès déplacé au Caire porte le numéro 1059 et celui apporté à Strasbourg le 1060.

[32] Lettre conservée aux Archives départementales du Bas-Rhin (Strasbourg), ADBR W1161/72.

[33] MONTET 1939, p. 76. 

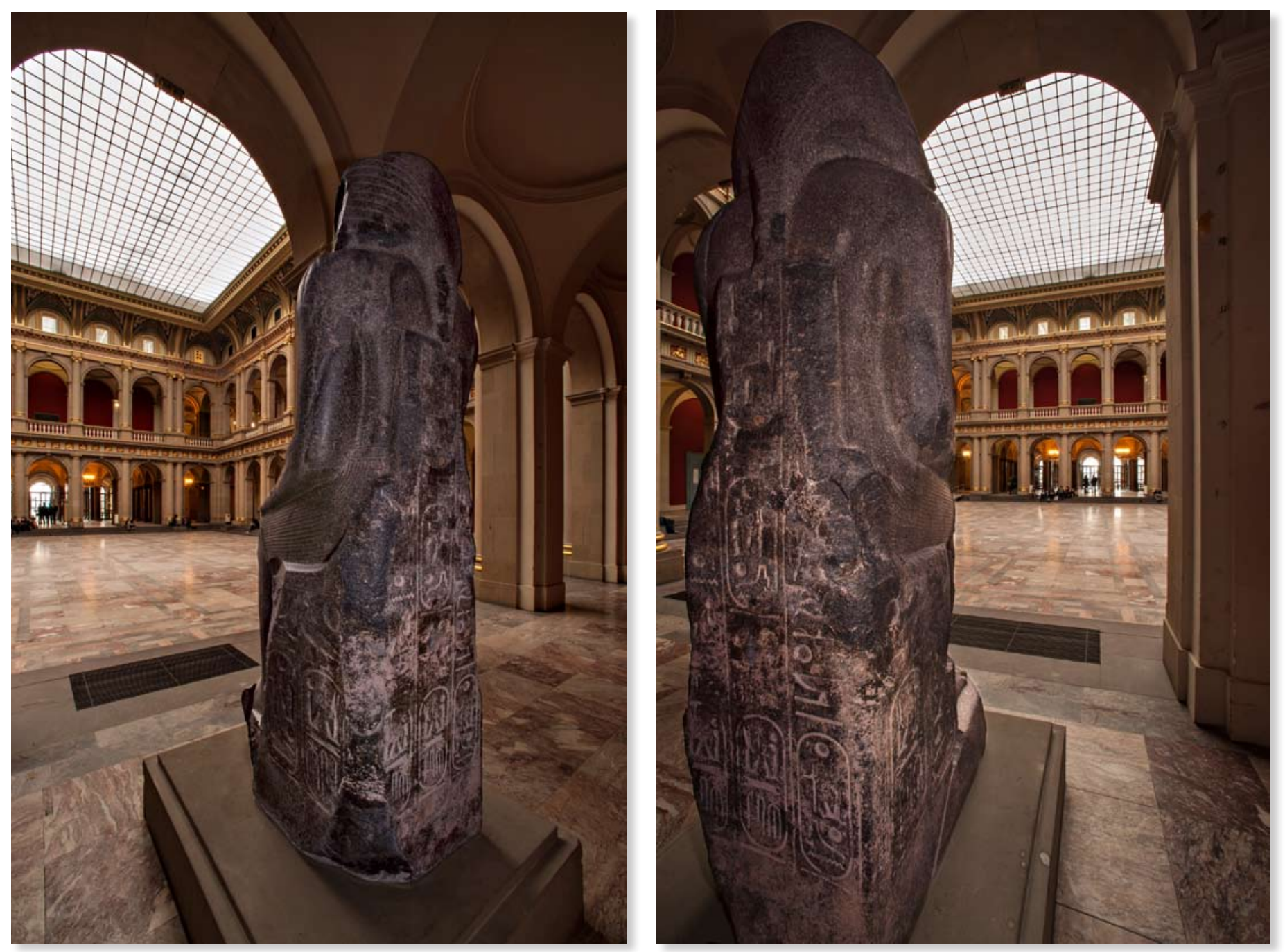

Figures $9 \mathrm{~b}$ et $9 \mathrm{c}$

Statue « maussade » de Ramsès II aujourd'hui au Palais Universitaire (๑ Pascal Disdier/CNRS)

Ramsès II soit installé au Palais du Rhin [34], mais finalement le Palais universitaire I'accueillera: «M. le recteur Dresch et le Conseil de I'Université ont voulu offrir à un aussi grand roi qui venait de si loin, une place digne de lui, au fond de l'aula, face à l'entrée » (MonTET 1939). Elle ornera ensuite l'entrée de l'Institut d'égyptologie (alors au rez-de-jardin) à partir des années soixante, et sera redéplacée en 1991 dans I'aula du Palais Universitaire, où elle se trouve toujours.

Il est possible d'établir une autre connexion entre les plaques de verre et la collection d'objets égyptiens de Strasbourg. En effet, sur le cliché TN 5/4 [35] (fig. 10) est photographiée une cinquantaine de figurines en

[34] Plusieurs travaux sur l'histoire du Palais du Rhin signalent que la collection égyptologique de l'université est conservée au Palais du Rhin après la Première Guerre Mondiale, en s'appuyant sur l'essai de LOHNER 1977, qui ne cite aucune de ses sources.

[35] Alb. MM 14, 35.

[36] Montet 1933a, p. 115-116 ; Montet 1942, p. 201. faïence d'Isis allaitante, complètes ou fragmentaires. Montet décrit à plusieurs reprises [36] le lot auquel appartiennent ces objets, trouvé dans le vestibule du temple dit d'Anta en 1930 [37], « au pied » d'une statue de Sekhmet et de Ramsès II. Cet ensemble de deux cents artefacts comprend deux plaquettes au nom de Siamon (identifiées dans la collection [38]), des bronzes [39], une stèle, trois fragments de statuettes et une cinquantaine de figurines d'Isis allaitante. P. Montet signale qu'à l'exception de l'inscription, ce lot est conservé à I'Institut d'égyptologie de Strasbourg [40]. L'une des Isis allaitantes représentées sur la plaque de verre est facilement identifiable grâce au serpent situé en bas du siège sur

[37] MONTET 1930, p. 7.

[38] IES_NI_1211.

[39] P. Montet ne les dénombre pas dans ces publications. Nous savons que le lot en comptait au moins sept, cités et photographiés dans MonTET 1933a, PI. LXIV, et LXV.

[40] MonTET 1942, p. 201. 
lequel Isis (dont le corps a disparu) était assise. Cette caractéristique a permis de retrouver facilement dans la collection strasbourgeoise cet objet (fig. 11-12), qui ne comportait aucun numéro d'inventaire antérieur à celui attribué en 2009 et dont la provenance était donc perdue jusqu'à ce jour [41]. Le recoupement effectué entre les objets photographiés sur plaques de verre et les objets strasbourgeois permettrait de systématiser l'identification des figurines, par exemple pour ces dizaines d'Isis allaitantes sans provenance connue présentes dans la collection.

L'intérêt scientifique de la collection de plaques de verre de Tanis est donc évident : dans la mesure où P. Montet publia seulement des descriptions succinctes de
I'assemblage découvert au temple de Mout et de Khonsou (dit temple d'Anta), I'étude croisée de la documentation photographique et des objets conservés à I'Institut d'égyptologie de Strasbourg permettrait d'établir les identifications nécessaires à un projet de nouvelle publication du lot, pour porter à la connaissance des chercheurs cet ensemble peu connu et mal documenté. De plus, cette étude permettrait d'approfondir les connaissances sur la collection de I'Institut d'égyptologie de Strasbourg, dont I'inventaire est en cours.

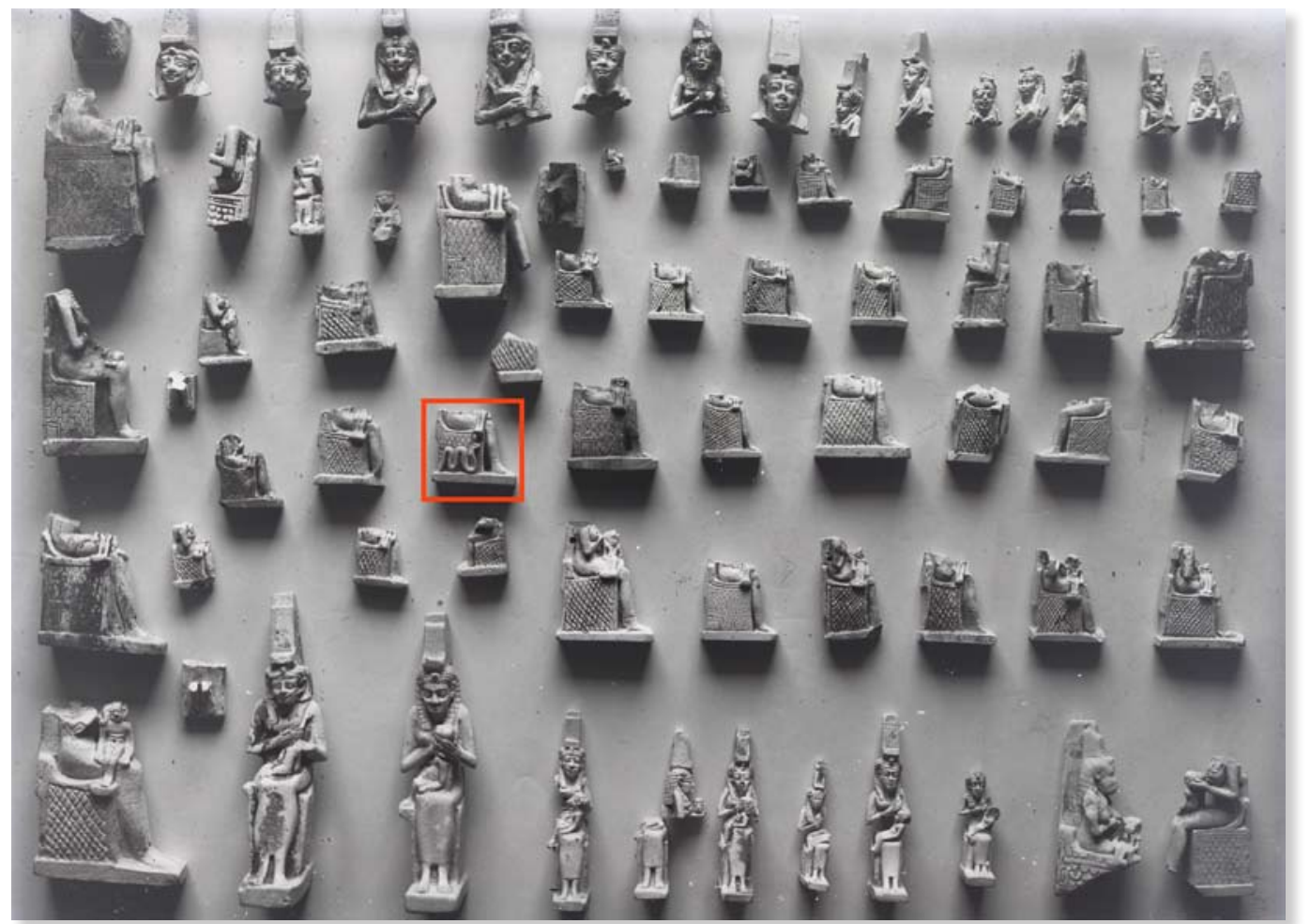

Figures 11 et 12
Figure 10 : IES Tn 5/4

Figurines en faïence siliceuse égyptienne d'Isis allaitante trouvées dans le temple dit d'Anta en 1930

Figurine en faïence siliceuse égyptienne d'Isis allaitante dans la collection de l'Institut d'égyptologie de Strasbourg IES_NI_0177 (cliché Fr. Colin)
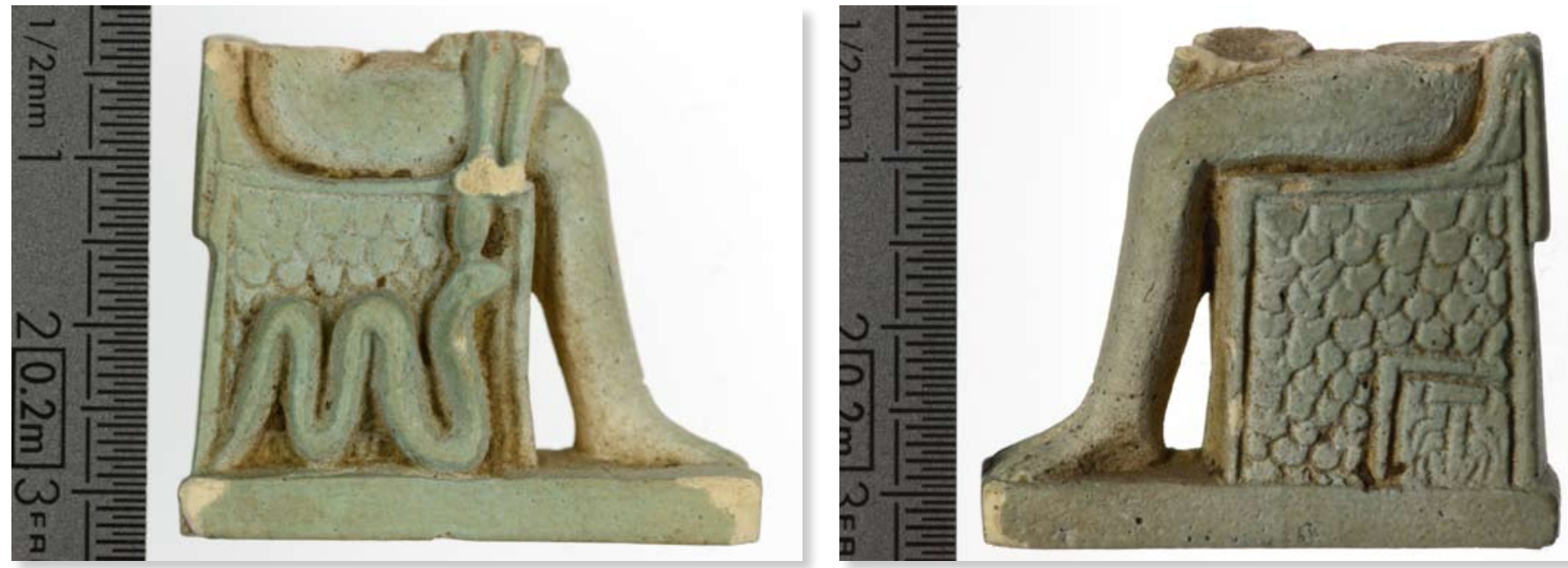
Brissaud, Philippe, Chauvet, Violaine \& HaIry, Isabelle, 1998, « Deux siècles de fouilles à Tanis : analyse des différents modes d'intervention sur le site», dans Philippe Brissaud et Christiane Zivie-Coche (éd.), Tanis. Travaux récents sur le Tell elHagar 1987-1997, Paris, p. 71-100.

Brissaud, Philippe, 2011, « Tanis : la nouvelle histoire du temple de Mout »,Égypte, Afrique et Orient 63, p. 73-80.

Brissaud, Philippe \& Desbordes, Christelle, 2012, « Aspects des temples de Tanis. L'exemple du temple de Mout », Égypte, Afrique et Orient 68, p. 25-34.

Le Guilloux, Philippe, 1998, «La Nécropole Royale de Tanis et les archives de la Mission Montet », dans dans Philippe Brissaud et Christiane Zivie-Coche (éd.), Tanis. Travaux récents sur le Tell el-Hagar 1987-1997, Paris, p. 179-192.

Leclere, François, 2006, Les villes de Basse-Égypte, Le Caire.

LoHNer, Jean-Daniel, 1977, Le palais du Rhin, I'ancien Kaiserpalast, Strasbourg, p. 6.

Montet, Pierre, 1930, «Les fouilles de Tanis en 1930 », BFL (S) IX, 1, p. 7.

Montet, Pierre, 1933a, Les nouvelles fouilles de Tanis (1919-1932), Paris.

Montet, Pierre, 1933b, «Les fouilles de Tanis en 1933 », BFL (S) XII, 1, p. 2-8.

Montet, Pierre, 1935-1938, « Les statues de Ramsès II à Tanis », dans Mélanges Maspéro, Le Caire, p. $498-508$.

Montet, Pierre, 1939, « Transport à Strasbourg d'une statue de Ramsès II », BFL (S) XVII, 3, p. 76.

Montet, Pierre, 1942, Tanis. Douze années de fouilles dans une capitale oubliée du Delta égyptien, Paris.

Montet, Pierre, 1998, Lettres de Tanis 1939-1940. La découverte des trésors royaux, Lettres éditées par Camille MontetBeaucour et Jean Yoyotte, Paris.

Yoyotre, Jean, 1965, « Reprise des fouilles de Tanis (avril-mai 1965) », CRAI 1965/2, p. 391-398. 